\title{
Entwicklungslinien für das Management alpiner Naturgefahren im Klimawandel (Essay)
}

\author{
Klaus Pukall Lehrstuhl für Wald- und Umweltpolitik, Technische Universität München (DE)* \\ Sylvia Kruse Eidgenössische Forschungsanstalt für Wald, Schnee und Landschaft (CH)
}

\begin{abstract}
Direction for the management of alpine hazards in times of climate change (essay)
Adapting to climate change means not only having to consider changing climate parameters but also to actively deal with societal change. Natural hazard management must therefore get involved in regional deliberation processes regarding future societal developments and consider these developments during the planning process.
\end{abstract}

Keywords: climate adaptation, natural hazard management, integrative risk management, Alpine space doi: $10.3188 /$ szf.2014.0037

*Hans-Carl-von-Carlowitz-Platz 2, DE-85354 München, E-Mail klaus.pukall@tum.de

$\mathrm{I}$ n der Berichterstattung über den Klimawandel und seine Folgen zeichnen die Medien und einige Wissenschaftler häufig Katastrophenbilder auch für Europa: Aufgrund des Anstiegs des Meeresspiegels werden küstennahe Gebiete überflutet, im Alpenraum werden ganze Talschaften durch Auftauen des Permafrosts und Ausbrechen von Gletscherseen unbewohnbar. In der schweizerischen Klimaanpassungsstrategie von 2012 wird für den Sektor «Umgang mit Naturgefahren» dagegen der Klimawandel nur als einer der Prozesse genannt, die die bestehende Gefährdung verstärken. Dementsprechend wird kein grosser Bedarf zur Anpassung an den Klimawandel festgestellt. Eine konsequente Umsetzung der Strategie der Nationalen Plattform Naturgefahren (PLANAT) und des integralen Risikomanagements würden ausreichen, um die Herausforderungen infolge des Klimawandels zu meistern (Schweizerischer Bundesrat 2012: 3808). Statt Katastrophenbewältigung, so auch der Grundtenor der Klimaanpassungsstrategien von Österreich und Deutschland, reiche routiniertes Handeln aus.

In diesem Essay vertreten wir eine vermittelnde Position. Wir gehen davon aus, dass die regionale Raumnutzung aufgrund notwendiger Massnahmen des Klimaschutzes und der Anpassung an den Klimawandel verstärkten Veränderungsprozessen unterworfen ist. Entsprechend muss das Naturgefahrenmanagement (NGM) im Alpenraum neben der Veränderung der klimatischen Bedingungen auch die Veränderung der Raumnutzung und des gesell- schaftlichen Risikoverständnisses berücksichtigen. Anpassung an den Klimawandel beinhaltet damit nicht nur die Suche nach robusten, flexiblen No-regret-Massnahmen, ${ }^{1}$ sondern verlangt im NGM eine Veränderung des Problemverständnisses, welches neben den sich verändernden Naturprozessen auch die sich wandelnde Gesellschaft umfassen muss.

Wir möchten daher aus unserer Sicht mögliche und sinnvolle Entwicklungslinien für das NGM aufzeigen. Wir kombinieren dabei normative Überlegungen, die auf der Überzeugung fussen, dass gesellschaftliche Entwicklungen im NGM bislang zu wenig berücksichtigt werden, mit empirischen Ergebnissen des interdisziplinären, vom deutschen Bundesministerium für Bildung und Forschung geförderten Forschungsprojekts «Alpine Naturgefahren im Klimawandel». ${ }^{2}$ In diesem untersuchen die beteiligten geistes- und sozialwissenschaftlichen Forscher, wie sich der Umgang mit Naturgefahren im Alpenraum im Verlauf der vergangenen Jahrhunderte verändert hat und ob sich das NGM im Alpenraum in jüngster Zeit durch den Diskurs über die Auswirkungen des

\footnotetext{
1 Diese Begriffe haben sich im Zuge der Klimaanpassungsdiskussion etabliert. Robuste Massnahmen können in einem breiten Bereich von Klimabedingungen ohne wesentliche Funktionseinbussen bestehen. Flexible Massnahmen zeichnen sich durch die Möglichkeit aus, dass sie immer an wechselnde Klimaprognosen angepasst werden können. Durch das Ergreifen von No-regret-Massnahmen sollen im Idealfall keine (finanziellen) Nachteile entstehen, auch wenn die erwartete Klimaänderung nicht eintritt.

2 http://alpine-naturgefahren.de (24.12.2013)
} 


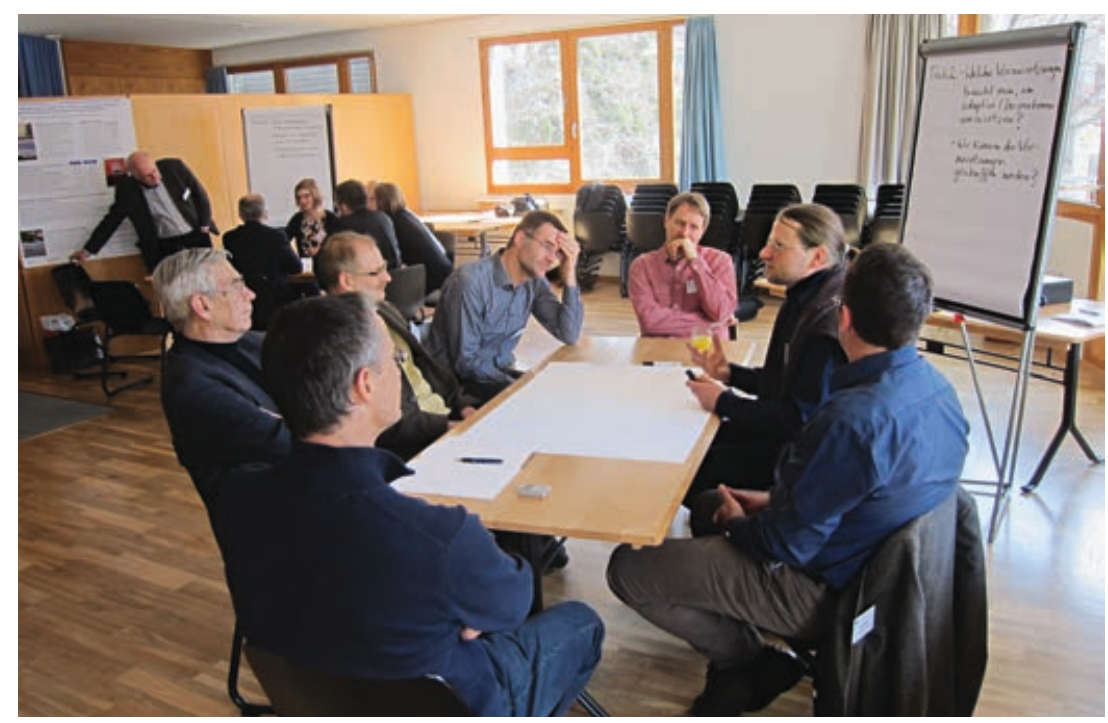

Abb 1 Internationaler Expertenworkshop im Forschungsprojekt «Alpine Naturgefahren im Klimawandel». Foto: Stefan Riesen

Klimawandels in einem Transformationsprozess befindet. Verglichen wird dabei das NGM in den Regionen (Ländern) Graubünden (Schweiz), Paznaun (Österreich) und Oberallgäu (Deutschland). Diesem Essay liegen Ergebnisse der Forschung zugrunde, insbesondere einer Inhaltsanalyse der Anpassungsstrategien und der vorbereitenden Dokumente, der Analyse von Interviews mit Experten des NGM aus Österreich, Deutschland und der Schweiz sowie des im Jahr 2013 durchgeführten internationalen Expertenworkshops «Adaptives NGM - passende Massnahmen für angepasste Organisationen in Zeiten des Klimawandels» (Abbildung 1).

\section{Naturgefahrenmanagement als dynamisches Feld}

Das NGM ist ständigem Anpassungsdruck ausgesetzt und verändert sich entsprechend laufend. Die beteiligten Akteure (z.B. Behörden, Gemeinden, Feuerwehren, Versicherer), so unsere These, sammeln dadurch Erfahrungen, die ihnen auch bei zukünftigen Veränderungen, zum Beispiel ausgelöst durch den Klimawandel, nützlich sein können. Neben unerwarteten und extremen Schadenereignissen sowie der wissenschaftlichen und technologischen Fortentwicklung möglicher Schutzmassnahmen prägen der Philosophiewandel innerhalb des NGM und der Einstellungswandel der Bevölkerung hinsichtlich Natur und Naturgefahren das NGM von heute:

1. Seit den 1970er-Jahren nehmen die Bemühungen des Schutzes einer möglichst unberührten Natur zu. Darauf wurde im NGM ab den 1980er-Jahren mit einer «Ökologisierung» vorab im Flussbau reagiert (Zaugg Stern 2006).

2. Angestossen von Vertretern der Wissenschaft und der Verwaltungen findet derzeit im NGM ein Philosophiewandel von der Gefahrenabwehr hin zum integralen Risikomanagement statt. Während in Deutschland und Österreich dieser Wandel bisher weitgehend nur die Kommunikation über Naturgefahren beeinflusst, orientiert sich die Managementpraxis in der Schweiz an diesem Leitbild. Beispiele dafür sind die Priorisierungen des Mitteleinsatzes mithilfe der Programmvereinbarungen des Bundes, bundeseinheitlicher Kartierungen (Silvaund Aquaprotect) und der Kosten-Nutzen-Abwägung auf Basis des Tools «EconoMe».

3. Ein zunehmender Teil der Bevölkerung ist nicht mehr oder kaum noch bereit, Schäden durch die Natur in Form von Naturgefahren in Kauf zu nehmen. Während es in den 1950er- und 1960er-Jahren noch selbstverständlich war, dass die Betroffenen im Katastrophenfall das Schadenausmass selbstständig zu begrenzen versuchten, wird heute erwartet, dass diese Aufgabe die staatlichen Akteure des Katastrophen- oder Zivilschutzes übernehmen. Zugespitzt formuliert, ist das NGM im letzten Jahrhundert zu erfolgreich geworden. In der Bevölkerung herrscht aufgrund der vielfach erfolgreichen Bewältigung von kleineren und mittleren Schadenereignissen ein grosses Vertrauen in das NGM. Wer hinter einem gut gepflegten Hochwasserdeich lebt, geht davon aus, dass er sicher ist, und macht sich berechtigterweise keine Gedanken über ein Ereignis, das vielleicht nicht einmal sein Enkelkind erleben wird.

\section{Klimawandel als zusätzlicher Faktor im Naturgefahrenmanagement}

Erste Überlegungen zum Umgang mit dem Klimawandel im NGM gehen auf die 1990er-Jahre zurück. So äusserte die Bayerische Wasserwirtschaftsverwaltung bereits 1992 die Befürchtung, dass die Schutzfähigkeit der Wälder nicht nur aufgrund des Waldsterbens, sondern auch aufgrund des Klimawandels abnehmen könnte (Oberste Baubehörde in Bayern 1992). Nach der Jahrtausendwende wurden dann erste Anpassungsstrategien (z.B. in Graubünden und Bayern) auf Basis der in den Ländern durchgeführten Forschungsprojekte und Studien (z.B. das schweizerische nationale Forschungsprogramm NFP 31 «limaänderungen und Naturkatastrophen») formuliert. Einigkeit besteht derzeit bezüglich der Annahme, dass sich im Zuge des Klimawandels vor allem fluviatile und geogene Prozesse verschärfen werden. Gleichzeitig herrscht aber auch grosse Unsicherheit darüber, wie sich der Klimawandel genau auf die Gefahrenprozesse auswirken wird. So ist beispielsweise die Prognose der Niederschlagsveränderungen, die für die Naturgefahren von zentraler Bedeutung sind, deutlich weniger belastbar als die der Temperaturerhöhung (C2SM 2011).

Für die Anpassung an den Klimawandel reicht es jedoch nicht aus, nur die Veränderungen der 
physikalischen Gefahrenprozesse zu betrachten. Auch gesellschaftliche Veränderungen spielen eine entscheidende Rolle. Zusätzlich zum «normalen» Flächenverbrauch lösen Massnahmen der Anpassung an den Klimawandel und des Klimaschutzes grundlegende Veränderungen der Landnutzung, insbesondere im Tourismus- und Energiesektor, aus. Der Verlust der notwendigen Schneesicherheit in tief gelegenen Wintersportgebieten wird auf der einen Seite zu verstärkten Ausbaubemühungen in den schneesicheren Regionen führen und auf der anderen Seite zur Neuausrichtung der Tourismuskonzepte in den tiefer gelegenen Destinationen. Dies kann wie in Immenstadt im Allgäu zur Aufgabe der Skilifte führen oder zur Schaffung neuer Infrastrukturen, um vom Skisport unabhängigen Tourismus zu fördern. Der Übergang wird dabei nicht gesteuert und einheitlich erfolgen, vielmehr werden - beeinflusst durch neue Trends - in regionalen Tourismuskonzepten und einzelbetrieblichen Entscheidungen unterschiedlichste Entwicklungspfade beschritten (Kruse et al 2013).

Im Energiesektor besteht inzwischen durch den politisch gewünschten Ausbau der erneuerbaren Energien ein viel höherer Flächenbedarf für den Anbau von nachwachsenden Rohstoffen und für Standorte von Windenergie-, Wasserkraft- sowie Fotovoltaikanlagen. Dies kann auf der einen Seite zu einer Erhöhung des Schadenpotenzials führen, auf der anderen Seite - zum Beispiel in Zusammenhang mit dem Ausbau der Wasserkraft - auch zu einer Verbesserung des Hochwasserschutzes.

\section{Entwicklungslinien für das Natur- gefahrenmanagement}

Unsere Experteninterviews zeigen, dass die Akteure des NGM bereits viele unterschiedliche Mass-

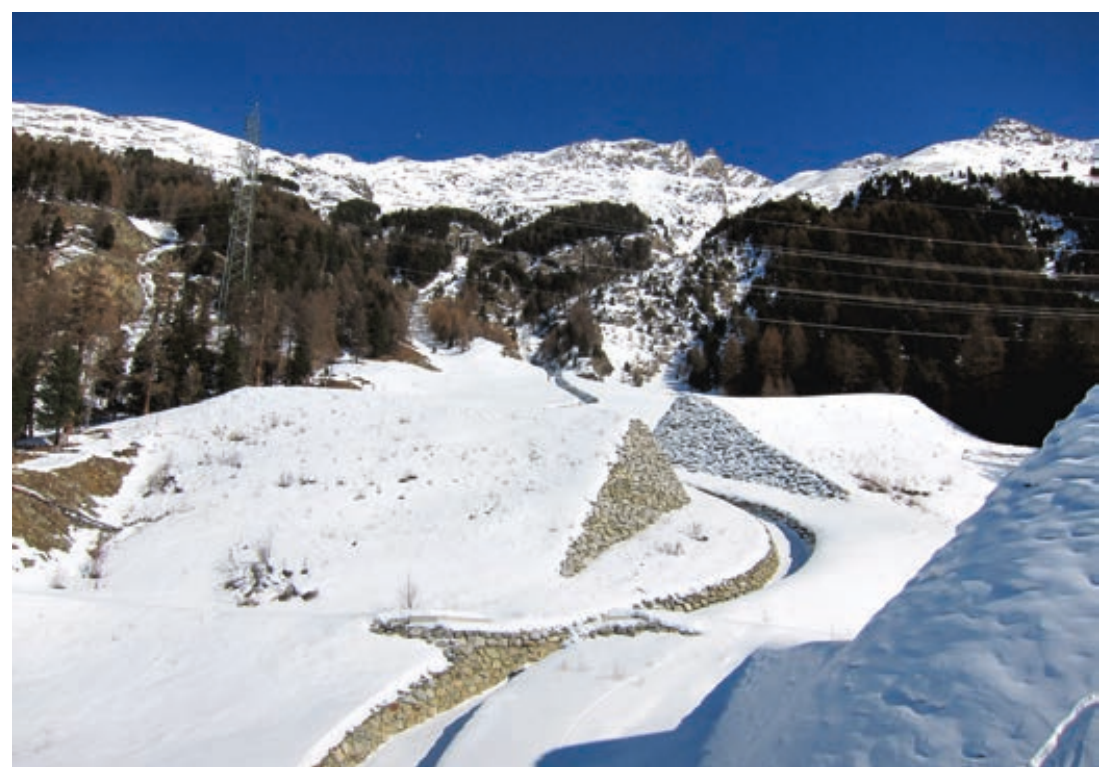

Abb 2 Auffangdämme als Anpassung an den auftauenden Permafrost in Pontresina. Foto: Sylvia Kruse nahmen umsetzen, die den Klimawandel mehr oder minder explizit berücksichtigen. Um jedoch die im Zuge des Klimawandels ablaufenden gesellschaftlichen Veränderungen aktiv mitgestalten zu können, sollte sich das NGM in dreierlei Hinsicht weiterentwickeln. Im Folgenden erläutern wir diese drei Entwicklungslinien.

\section{Von der Analyse der Vergangenheit zur Orientierung an der Zukunft}

Das für das NGM grundlegende Konzept der Jährlichkeit ist auf eine Betrachtung möglichst langer historischer Zeitreihen ausgelegt. Je länger die Beobachtung zurückreicht, desto besser kann das Magnitude-Frequenz-Verhältnis der betrachteten Gefahren bestimmt werden. Dieser Vergangenheitsbezug wird im Rahmen der Anpassung an den Klimawandel durch Zukunftsszenarien ergänzt. Regionale Klimaszenarien dienen inzwischen als Grundlage für neue Schutzkonzepte. Die Einführung eines einheitlichen Klimaänderungsfaktors von 15\% (d.h., das aus historischen Daten errechnete Bemessungsereignis wird um den Faktor 1.15 erhöht) für Hochwasserschutzbauten in Bayern im Jahr 2004 oder der 2003 fertiggestellte Lawinen- und Murgangschutzdamm in Pontresina (Graubünden) sind Ausdruck der an zukünftigen Gefahren orientierten Vorgehensweise.

Diese Zukunftsorientierung sollte jedoch nicht auf Prozesse im Naturraum beschränkt bleiben, sondern auf die sozialen Prozesse ausgeweitet werden. Bei der Wirtschaftlichkeitsbetrachtung verschiedener Schutzkonzepte (z.B. durch EconoMe) unterbleibt dies derzeit. Das Kosten-Nutzen-Verhältnis wird auf Basis des bestehenden Schadenpotenzials errechnet. Die zukünftigen Veränderungen, die durch die Umsetzung der Schutzkonzepte ermöglicht beziehungsweise ausgelöst werden, fliessen nicht systematisch in die Betrachtung ein. Nach dem Bau von technischen Schutzmassnahmen ist normalerweise eine verstärkte Siedlungstätigkeit in den geschaffenen «sicheren» Räumen festzustellen (Burby 2006; Abbildung 3). Durch die Erhöhung des Schadenpotenzials wird somit der Sicherheitsgewinn langfristig wieder zunichtegemacht. Das Gesamtrisiko sinkt unmittelbar nach der Baumassnahme deutlich ab, steigt aber langfristig teilweise sogar über das Ursprungsniveau hinaus. Dieser Effekt ist auch bei der Gefahrenzonenplanung beobachtbar. Mithilfe einer Betrachtung der historischen Entwicklung der Baugebiete in Galtür (Paznaun) und Davos (Graubünden) konnten Keiler (2004) und Fuchs et al (2004) zeigen, dass das Schadenpotenzial an den Zonengrenzen die grösste $\mathrm{Zu}$ nahme erfuhr. Hier war ja gerade von Behördenseite gezeigt worden, dass der Raum sicher ist und somit einer Siedlungsentwicklung nichts entgegensteht.

In den alpinen Gebieten, in denen vor Naturgefahren geschützte Räume ein knappes Gut sind, 


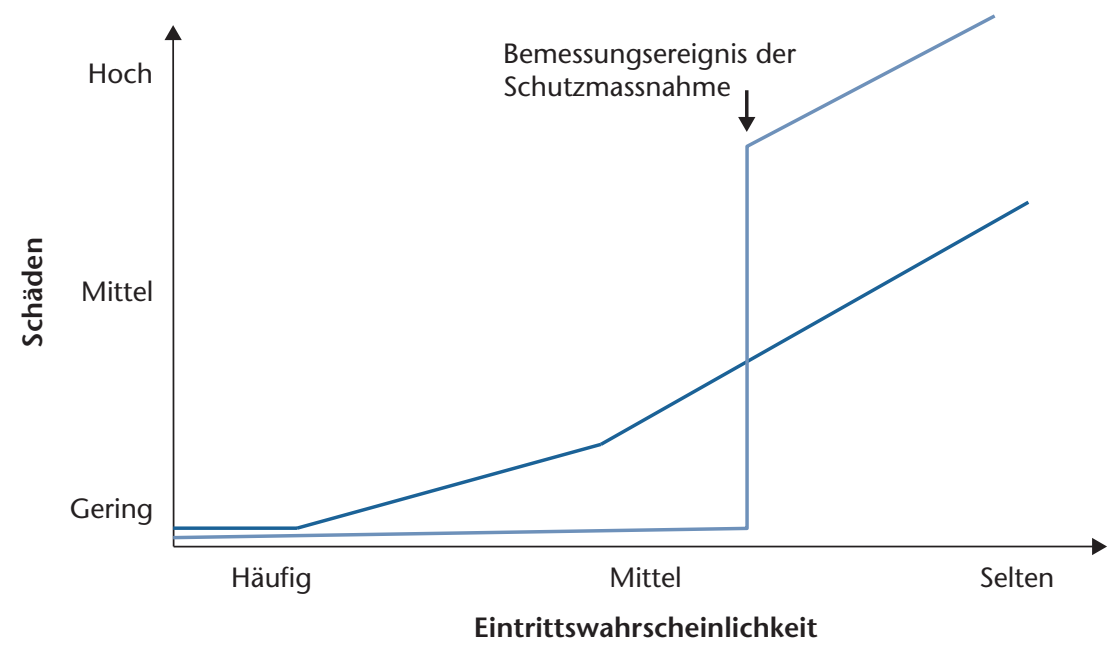

- Ohne technische Schutzmassnahmen _ Mit technischen Schutzmassnahmen

Abb 3 Das Safe-Developement-Paradox in der Siedlungsentwicklung nach Burby (2006). Durch die Errichtung von technischen Schutzmassnahmen entstehen deutlich seltener Schäden durch Naturgefahren. Die Schadensummen im Schadenfall sind aber wegen einer fehlenden Gefahrenwahrnehmung der Bewohner der "sicheren" Gebiete und einer langfristig stärkeren Akkumulation von Schadenpotenzial höher.

ist die beschriebene Entwicklung des Schadenpotenzials explizit oder implizit erwünscht. Wilhelm (1999) argumentiert dementsprechend, dass neben dem Naturgefahrenrisiko auch die Chancen, die durch die Nutzung der gefährdeten Räume entstehen, betrachtet werden sollten. Aus volkswirtschaftlicher Sicht ist es sinnvoll, nicht nur die absolut sicheren Gebiete zu besiedeln.

In Planungsprozessen sollten also in Zukunft nicht nur Klimaszenarien, sondern auch regionale und lokale Raumentwicklungsszenarien systematisch berücksichtigt werden, um einen optimalen Einsatz der staatlichen Mittel zum Schutz vor Naturgefahren zu gewährleisten.

\section{Von der Kartierung der Naturgefahren zur Aushandlung der Raumentwicklung}

Viele Akteure des NGM betrachten die Zunahme von Infrastrukturwerten und die Bebauung von potenziell gefährdeten Gebieten als die zentrale Ursache für den Anstieg der Naturgefahrenrisiken. Die betrachteten Länder setzten zur Begrenzung dieser nicht erwünschten Entwicklung überwiegend auf eine Top-down-Strategie (Wagner 2009): Durch die Ermittlung von Gefahrenzonen (Österreich, Schweiz) beziehungsweise Überschwemmungsgebieten (Deutschland) werden gefährdete von ungefährdeten Gebieten fachplanerisch getrennt. Auf nationaler beziehungsweise kantonaler Ebene wird dabei ein verbindliches Sicherheitsniveau (z.B. rote oder gelbe Zone) festgelegt. Die für die lokale Raumplanung zuständigen Gemeinden können sich in der Regel nicht über diese Fachplanung hinwegsetzen, es besteht für sie kaum ein planerischer Ermessensspielraum. Durch dieses Vorgehen soll gewährleistet werden, dass die Naturgefahren in einem politisch gewollten Mindestmass bei allen Planungen berücksichtigt werden. Selbst starke gesellschaftliche Interessen sollen sich nicht einfach über die gesetzten Mindestgrenzen hinwegsetzen können. Die Gefahrenzonenplanung trifft aber wegen dieses Top-downVorgehens auf Widerstände der lokalen Akteure (Schmid 2010). Diese akzeptieren die Gefahrenkartierungen aufgrund ihrer abweichenden eigenen Gefahrenwahrnehmung zum Teil nicht und berücksichtigen daher die Gefahren nur unzureichend bei ihren eigenen Planungsentscheidungen. In vielen Alpengemeinden besteht daher die Tendenz, sich möglichst nah an den Gefahrenraum heran zu entwickeln, anstatt nach möglichst sicheren Bauplätzen zu suchen.

Wegen des starken Fokus auf die Gefahrenzonenplanung ist die raumplanerische Auseinandersetzung über erwünschte oder erwartete Entwicklungsprozesse, bei welcher die Beschränkungen, aber auch Möglichkeiten des «Gefahrenraums» beachtet werden, äusserst selten (Höferl 2010). Dies liegt sicherlich auch an der in den drei Alpenländern geringen Bedeutung der regionalen und überregionalen Raumplanung: Der Raumplanung stehen (im Vergleich zur Fachplanung des NGM) wenig personelle und finanzielle Ressourcen zur Verfügung, ausserdem weisen die überörtlichen Pläne nur eine geringe Verbindlichkeit auf (Kruse \& Pütz 2014).

Aushandlungsprozesse über die lokale und regionale Raumnutzung, das gewünschte Landschaftsbild, das Tourismuskonzept und auch die notwendigen Massnahmen zur Anpassung an den Klimawandel laufen daher häufig informell ab und werden erst nach möglicherweise schon weitgehenden Vorentscheidungen in formale Planungs- oder Genehmigungsprozesse eingespeist. Die staatlichen Träger öffentlicher Belange wie die Forst- und Wasserbauverwaltungen können somit erst spät mit ihren Vorstellungen zum Schutz vor Naturgefahren die Ideenentwicklung beeinflussen. Sie finden sich daher häufig in der Rolle der Verhinderer wieder, oder es wird Druck ausgeübt, Projekte der Regionalentwicklung mithilfe von technischen Schutzmassnahmen zu ermöglichen.

Aus unserer Sicht sollten sich alle Akteure des NGM aktiv in die lokalen Raumentwicklungsprozesse einbringen. Eine zentrale Rolle könnten dabei die Mitglieder der Lawinen- beziehungsweise Gefahrenkommissionen, der Feuerwehren sowie die in der Schweiz neu geschaffenen Naturgefahrenberater spielen. Alle diese Akteure sind gut in die lokalen Netzwerke eingebunden und kommen somit frühzeitig mit neuen Entwicklungsideen in Kontakt. Auf der einen Seite könnten sie im Zuge der Ausbildung dafür sensibilisiert werden, dass sie Multiplikatoren für das Thema Naturgefahren sind und somit nicht nur für ihr unmittelbares Aufgabenfeld (Katastrophenschutz und Warnung) sprechen sollten. Auf der 


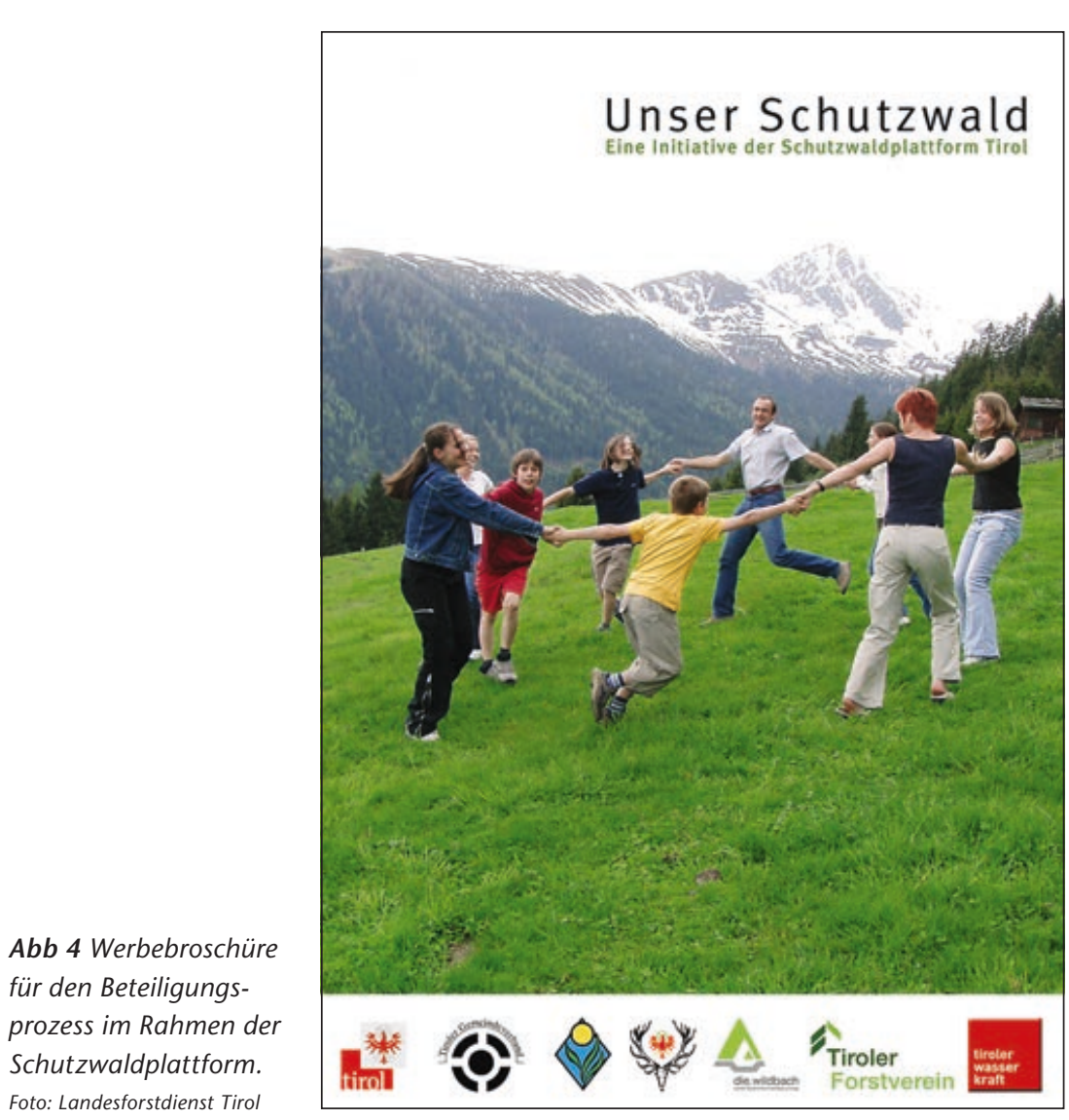

werden inzwischen Flussraummanagementpläne erstellt. Hierbei werden interdisziplinär alle Raumansprüche bezüglich des betrachteten Flusses erhoben und innerhalb des Flussraumforums diskutiert. Am Flussraumforum beteiligen sich neben den staatlichen Ämtern alle betroffenen Gemeinden und Vertreter aller Wassernutzer. Erste Erfahrungen zeigen, dass mithilfe dieses Beteiligungsprozesses ein gemeinsames Verständnis für den Risiko- und Nutzungsraum entsteht sowie das Vertrauen und die Zusammenarbeit zwischen den beteiligten Akteuren verbessert werden. Damit wird die Basis für eine gemeinsame Risikokultur gelegt. Nachteilig sind die aufwendigeren und langwierigeren Entscheidungsprozesse sowie die teilweise fehlenden Ressourcen für die Umsetzung der vereinbarten Massnahmen. ${ }^{4}$

Aus unserer Sicht sollten diese aushandlungsorientierten Beteiligungsverfahren weiter ausgebaut werden. In ihnen können Zukunftsentwürfe und Leitbilder entwickelt werden, die nicht nur die gesamtgesellschaftlich gewünschten Mindeststandards des NGM berücksichtigen, sondern die unterschiedlichen Ansprüche der gesellschaftlichen Gruppen verknüpfen. Wie bereits erwähnt, sollten sich insbesondere die staatlichen Akteure des NGM verstärkt in formale und informale Beteiligungsprozesse einbringen, die von anderen Akteuren initiiert werden anderen Seite könnten sie als Mittler zu den staatlichen Behörden auftreten, sodass sich im Bedarfsfall die Behörden frühzeitig in informelle Planungen einbringen und damit im Diskussionsprozess den Naturgefahrenschutz stärken können.

\section{Von der Schaffung von Akzeptanz zur gemeinsamen Risikokultur}

Der Beteiligung lokaler Akteure an Planungen des NGM wurde in den letzten Jahren ein immer grösseres Gewicht beigemessen. Dies lässt sich zum Beispiel an der Neugestaltung des Finanzausgleichs zwischen Bund und Kantonen in der Schweiz verdeutlichen: Partizipativ geplante Projekte werden prioritär behandelt und erhalten $2 \%$ höhere Bundesbeiträge. Im Vordergrund stehen dabei Effektivitätsziele: Konflikte zwischen unterschiedlichen Interessen sollen bearbeitet, Akzeptanz für die Planung geschaffen und somit Verzögerungen bei der Realisierung von Projekten vermieden werden (BAFU 2008). Auch projektunabhängige Beteiligungsformen wurden bereits implementiert. Im Tirol etablierte der Forstdienst die sogenannte Schutzwaldplattform, um damit «Allianzen mit den für die Landschaftsnutzung befassten Institutionen zur Erhaltung und Verbesserung des Schutzwaldes» zu bilden. ${ }^{3}$ In diesem Zuge wurden auch Schutzwaldpartnergemeinden ausgezeichnet, in denen lokale Gruppen miteinander die Bevölkerung für das Thema Schutzwald sensibilisieren und gemeinsam Projekte im Schutzwald planen. Im Südtirol (Italien) (z.B. zur Neuausrichtung von Tourismuskonzepten oder der Wirtschaftsförderung).

\section{Notwendige Rahmenbedingungen}

Die im vorangehenden Kapitel dargestellten Entwicklungslinien des NGM stellen zum Teil eine Weiterentwicklung bestehender Prozesse, zum Teil eine Neuausrichtung dar. Um sie zu unterstützen, sind verschiedene Rahmenbedingungen notwendig: 1. Politische Rückendeckung: Wir empfehlen, dass sich das NGM aktiver in die Regionalentwicklung einbringt. Hierfür ist Rückendeckung auf den verschiedenen politischen Ebenen notwendig, die die Verhandlungsposition des NGM gegenüber den starken wirtschaftlichen Interessen stärkt.

2. Kommunikationsfähigkeit und Konfliktbereitschaft: Unabdingbare Voraussetzungen für die aktive Beteiligung in regionalen Aushandlungsprozessen sind die Fähigkeit und die Bereitschaft, mit den anderen zu kommunizieren und Konflikte offen auszuhandeln. Dafür müssen die Akteure des NGM nicht nur kommunikationsstark und konfliktbereit sein, sondern auch über genügend Zeit verfügen, um

\footnotetext{
3 https://www.tirol.gv.at/umwelt/wald/schutzwald/plattform/ swp-tirol/swplattformziele/ (18.12.2013)

4 HECHER P (2012) Flussraummanagement in Südtirol. Unveröffentlichter Vortrag anlässlich des Workshops «Bergwald und Naturgefahren - ein Fall für den Runden Tisch?!» am 19.1.2012 in Schaan (FL).
} 
sich in den Aushandlungsprozessen aktiv einbringen zu können.

3. Verhandlungsspielräume schaffen: Strikte nationale/kantonale Regulierungen (z.B. bezüglich der Rechtsfolgen von Gefahrenzonenplänen und der notwendigen Ausbaustandards für technische Verbauungen) sollten flexibilisiert werden, um Verhandlungsspielräume zu schaffen. So könnte sich selbst eine Bebauung in einer roten Lawinenzone als sinnvoll herausstellen, wenn ausschliesslich eine Nutzung in den Sommermonaten stattfindet und der Investor - beispielsweise aufgrund einer kurzen Amortisationszeit seiner Investition - bereit ist, das Risiko zu tragen.

«Wieso sollen wir uns mit der Anpassung an den Klimawandel beschäftigen? Wenn wir das integrierte Risikomanagement umsetzen, sind wir auch den Herausforderungen des Klimawandels gewachsen.» Diese anlässlich des Expertenworkshops geäusserte Feststellung, die sich mit ähnlichen Worten auch in der schweizerischen Klimaanpassungsstrategie wiederfindet, bedarf aus unserer Sicht folgender Präzisierung: Gerade die Diskussion über den Klimawandel verdeutlicht, dass wir für einen zielgerichteten Umgang mit Naturgefahren verschiedene mögliche Zukunftsszenarien mit bedenken müssen. Es reicht dabei nicht aus, nur die klimatischen Variablen zu betrachten. Die lokal und regional unterschiedliche Entwicklung der gesellschaftlichen Ansprüche an die Raumnutzung und des Risikoverständnisses bedürfen ebenfalls der Berücksichtigung.

Eingereicht: 19. Dezember 2013, akzeptiert (ohne Review): 20. Dezember 2013

\section{Literatur}

BAFU (2008) Handbuch NFA im Umweltbereich. Bern: Bundesamt Umwelt. 208 p.
BURBY RJ (2006) Hurricane Katrina and the paradoxes of government disaster policy: Bringing about wise governmental decisions for hazardous areas. Ann Am Acad Political Social Sci 604: 171-191.

C2SM (2011) Swiss climate change scenarios CH2011. Zürich: Eidg Techn Hochschule, Center for Climate Systems Modeling. $88 \mathrm{p}$.

FUCHS S, BRÜNDL M, STÖTTER J (2004) Development of avalanche risk between 1950 and 2000 in the municipality of Davos, Switzerland. Nat Hazards Earth Syst Sci 4: 263-275.

HÖFERL K (2010) Von der Gefahrenabwehr zur Risikokultur. Diskurse zum raumplanerischen Umgang mit Hochwasser in (Nieder-)Österreich. Wien: Univ Bodenkultur, Dissertation. $253 \mathrm{p}$.

KEILER M (2004) Development of the damage potential resulting from avalanche risk in the period 1950-2000, case study Galtür. Nat Hazards Earth Syst Sci 4: 249-256.

KRUSE S, PÜTZ M (2014) Adaptive capacities of spatial planning in the context of climate change in the European Alps. Eur Plan Stud. doi: 10.1080/09654313.2013.860516

KRUSE S, STIFFLER M, BAUMGARTNER D, PÜTZ M (2013) Vulnerability and adaptation to climate change in the alpine space: a case study on the adaptive capacity of the tourism sector. In: Schmidt-Thomé P, Greiving S, editors. European climate vulnerabilities and adaptation: a spatial planning perspective. Chichester: Wiley. pp. 273-288.

OBERSTE BAUBEHÖRDE IN BAYERN (1992) Wildbäche, Lawinen: Programm 2000. Staatsministerium Innern, Wasserwirtschaft Bayern 24. 124 p.

SCHMID FS (2010) Sichtweisen von lokalen Akteuren und Fachexperten in der Naturgefahrenprävention. Bern: Univ Bern, Geograph Inst, Geographica Bernensia G85. 186 p.

SCHWEIZERISCHER BUNDESRAT (2012) Anpassung an den Klimawandel in der Schweiz. Ziele, Herausforderungen und Handlungsfelder. Erster Teil der Strategie des Bundesrates vom 2. März 2012. BBI 2012: 3777-3858.

WAGNER K (2009) Konflikte bei der Festsetzung von Überschwemmungsgebieten: Die Schwierigkeit, bestehende Schutzstrategien zu verändern. Z Umweltpolit Umweltr 32: 93-115.

WILHELM C (1999) Naturgefahren und Sicherheit der Bevölkerung im Gebirge - oder: Von der Schicksalsgemeinschaft zur Risikogesellschaft. In: WSL. Nachhaltige Nutzung im Gebirgsraum. Birmensdorf: Eidgenöss Forsch.anstalt WSL, Forum Wissen. pp. 47-55.

ZAUGG STERN M (2006) Philosophiewandel im schweizerischen Wasserbau: Zur Vollzugspraxis des nachhaltigen Hochwasserschutzes. Zürich: Univ Zürich, Dissertation. 369 p.

\section{Entwicklungslinien für das Management alpiner Naturgefahren im Klimawandel (Essay)}

Anpassung an den Klimawandel bedeutet nicht nur die Berücksichtigung veränderter Klimaparameter, sondern eine aktive Auseinandersetzung über gesellschaftliche Entwicklungen. Das Naturgefahrenmanagement sollte sich daher stärker in regionale Aushandlungsprozesse über zukünftige Entwicklungen einbringen und diese gesellschaftlichen Entwicklungen auch bei der Planung berücksichtigen.

\section{Tendences de la gestion des dangers naturels alpins face au changement climatique (essai)}

S'adapter aux changements climatiques ne signifie pas seulement tenir compte de paramètres climatiques variables, mais également de débattre activement des développements sociaux. La gestion des dangers naturels devrait pour cette raison s'impliquer davantage dans les débats régionaux sur les développements futurs et également intégrer ces développements sociaux dans la planification. 